\title{
AUTHOR INDEX (Volume 10)
}

\author{
Abou Shakra, F., \\ Asymptotics of the \\ critical nonlinear wave \\ equation for a class of \\ non-star-shaped \\ obstacles
}

Aleksić, J. \& Mitrović, D., Strong traces for averaged solutions of heterogeneous ultra-parabolic transport equations

Almada, C., Decay rates for the shifted wave equation on a symmetric space of noncompact type

Bouchut, F. \& Crippa, G., Lagrangian flows for vector fields with gradient given by a singular integral

Candy, T., Bilinear estimates and applications to global well-posedness for the Dirac-Klein-Gordon equation on $\mathbb{R}^{1+1}$

Chae, M., Kang, K. \& Lee, J., Global classical solutions for a compressible fluid-particle interaction model

Charão, R. C., da Luz, C. R. \& Ikehata, R., New decay rates for a problem of plate dynamics with fractional damping

Chen, G., Young, R. \& Zhang, Q., Shock formation in the compressible Euler equations and related systems
3 (2013) 495

4 (2013) 659

4 (2013) 677

2 (2013) 235

1 (2013) 1

3 (2013) 537

3 (2013) 563

1 (2013) 149
Colliander, J., Czubak, M. \& Sulem, C., Lower bound for the rate of blow-up of singular solutions of the Zakharov system in $\mathbb{R}^{3}$

3 (2013) 523

Crippa, G., see Bouchut, F.

2 (2013) 235

Czubak, M., see Colliander, J.

3 (2013) 523

da Luz, C. R., see Charão, R. C.

3 (2013) 563

Dafermos, C. M., Hyperbolic systems of balance laws with weak dissipation II

1 (2013) 173

Dharmawardane, P. M. N., Global solutions and decay property with regularity-loss for quasi-linear hyperbolic systems with dissipation

Donatelli, D., The artificial compressibility approximation for $M H D$ equations in unbounded domain

1 (2013) 181

Dutta, R., A bounded variation estimate for the force scheme applied to strictly hyperbolic systems of conservation laws in the temple class

El Hajj, A. \& Monneau, R., Uniqueness results for diagonal hyperbolic systems with large and monotone data

1 (2013) 37

1 (2013) 105

3 (2013) 461

Frid, H., Risebro, N. H. \& Sande, H., Spatially periodic solutions for gas flows with pressure depending on a variable coefficient 
Garavello, M. \& Piccoli, B., Coupling of Lighthill-

Whitham-Richards and phase transition models

Goudiaby, M. S. \& Kreiss, G., A Riemann problem at a junction of open canals

Ikehata, R., see Charão, R. C.

Kang, K., see Chae, M.

Katayama, S. \& Kubo, H., Lower bound of the lifespan of solutions to semilinear wave equations in an exterior domain

Kreiss, G., see Goudiaby, M. S.

Kubo, H., see Katayama, $\mathrm{S}$.

Lee, H. \& Rendall, A. D., The Einstein-Boltzmann system andpositivity

Lee, J., see Chae, M.

LeFloch, P. G., Hyperbolic balance laws with entropy on a curved spacetime.

The weak-strong uniqueness theory

Li, T. \& Li, X., Asymptotic shapes of shock curves for some critical cases

Li, X., see Li, T.

Marchesin, D., see

Rodríguez-Bermúdez, P.

Mi, Y. \& Mu, C.,

Well-posedness and analyticity for an integrable two-component system with cubic nonlinearity

Mitrović, D., see Aleksić, J.

Monneau, R., see El Hajj, A.
Mu, C., see Mi, Y.

4 (2013) 703

Okamoto, M.,

Well-posedness of the

3 (2013) 577

Cauchy problem for the

Chern-Simons-Dirac

system in two

dimensions

3 (2013) 431

3 (2013) 563

3 (2013) 537

2 (2013) 199

3 (2013) 431

2 (2013) 199

1 (2013) 77

3 (2013) 537

4 (2013) 773

3 (2013) 415

3 (2013) 415

2 (2013) 335

Piccoli, B., see Garavello, M.

Rendall, A. D., see Lee, H.

Risebro, N. H., see Frid, H.

Roberts, J., Steady and self-similar full Euler flow

Rodríguez-Bermúdez, P. \& Marchesin, D., Riemann solutions for vertical flow of three phases in porous media: Simple cases

Rózga, K., On a family of convex solutions to a hyperbolic partial differential equation

Sande, H., see Frid, H.

Sulem, C., see Colliander, J.

Tarfulea, N., On boundary conditions for first-order symmetric hyperbolic systems with constraints

Wang, J. \& Yu, P., Long time solutions for wave maps with large data

Xiao, Q.-H. \& Zhao, H.-J., Nonlinear stability of planar stationary waves for generalized

Benjamin-Bona-

Mahony-Burgers equations in the half-plane
4 (2013) 703
4 (2013) 659
3 (2013) 461

Young, R., see Chen, G.

Yu, P., see Wang, J.

Zhang, Q., see Chen, G.

Zhao, H.-J., see Xiao, Q.-H.
4 (2013) 735

3 (2013) 577

1 (2013) 77

1 (2013) 129

4 (2013) 799

2 (2013) 335

4 (2013) 637

1 (2013) 129

3 (2013) 523

4 (2013) 725

2 (2013) 371

2 (2013) 283

1 (2013) 149

2 (2013) 371

1 (2013) 149

2 (2013) 283 